\title{
Kinetic inductance detectors for the Origins Space Telescope
}

\author{
Steven Hailey-Dunsheath, ${ }^{\mathrm{a}, *}$ Reinier M. J. Janssen ${ }^{\circ},{ }^{\text {a,b }}$ Jason Glenn, ${ }^{\mathrm{c}}$ \\ Charles M. Bradford, ${ }^{\mathrm{a}, \mathrm{b}}$ Joanna Perido $\odot,{ }^{\mathrm{d}}$ Joseph Redford, ${ }^{\mathrm{a}}$ and \\ Jonas Zmuidzinas ${ }^{\text {a }}$ \\ ${ }^{a}$ California Institute of Technology, Pasadena, California, United States \\ bJet Propulsion Laboratory, California Institute of Technology, Pasadena, California, \\ United States \\ ${ }^{c}$ NASA Goddard Space Flight Center, Greenbelt, Maryland, United States \\ ${ }^{\mathrm{d}}$ University of Colorado at Boulder, Department of Astrophysics and Planetary Sciences, \\ Boulder, Colorado, United States
}

\begin{abstract}
The Origins Space Telescope (Origins) will have a 5.9-m diameter primary mirror cooled to $4.5 \mathrm{~K}$ and will be equipped with three instruments, two of which will cover the far-IR $(\lambda=25$ to $588 \mu \mathrm{m})$. These far-IR instruments will require large arrays $\left(\sim 10^{4}\right.$ detectors $)$ of ultrasensitive detectors, with noise equivalent powers (NEPs) as low as $3 \times 10^{-20} \mathrm{~W} \mathrm{~Hz}^{-1 / 2}$. Kinetic inductance detectors (KIDs) have already demonstrated the array format, modularity, and readout multiplexing density requirements for Origins; the only aspect that requires improvement is the per-pixel sensitivity. We show how KIDs can meet the sensitivity target, focusing on two existing architectures that together demonstrate the key necessary attributes. Arrays of antenna-coupled coplanar waveguide resonators have achieved NEPs of $3 \times 10^{-19} \mathrm{~W} \mathrm{~Hz}^{-1 / 2}$ in laboratory demonstrations at $350 \mu \mathrm{m}$; they demonstrate excellent material properties as well as array-level integration and performance. Lumped element detectors such as those under development for balloon-borne spectroscopy at 10 to $350 \mu \mathrm{m}$ demonstrate flexibility in coupling to shorter-wavelengths, reducing active volume, and providing a means for suppressing capacitor noise. A straightforward combination of the elements of these already-demonstrated devices points to a low-volume design that is expected to meet the Origins sensitivity targets. (C) The Authors. Published by SPIE under a Creative Commons Attribution 4.0 Unported License. Distribution or reproduction of this work in whole or in part requires full attribution of the original publication, including its DOI. [DOI: 10.1117/1.JATIS.7.1.011015]
\end{abstract}

Keywords: kinetic inductance detectors; Origins Space Telescope; far-infrared.

Paper 20082SS received Jun. 15, 2020; accepted for publication Jan. 26, 2021; published online Mar. 18, 2021.

\section{Introduction}

The Origins Space Telescope (Origins) traces our cosmic history, from the formation of the first galaxies and the rise of metals to the development of habitable worlds and present-day life. Origins does this through exquisite sensitivity to infrared radiation from ions, atoms, molecules, dust, water vapor, and ice, and observations of extra-solar planetary atmospheres, protoplanetary disks, and large-area extragalactic fields. Origins operates in the wavelength range 2.8 to $588 \mu \mathrm{m}$ and is more than 1000 times more sensitive than its predecessors due to its large, cold (4.5 K) telescope and advanced instruments.

Origins will have two instruments operating at $\lambda>25 \mu \mathrm{m}$, a broadband imager (Far-IR Imager and Polarimeter; FIP, Staguhn et al., this volume) and an $R=300$ spectrometer (Origins Survey Spectrometer; OSS, Bradford et al., this volume). The significant improvement over the scientific capabilities of prior far-IR missions is based on the cold telescope $(4.5 \mathrm{~K})$ combined with low-noise far-IR detectors. The detector noise equivalent power (NEP) targets for imaging and spectroscopy are $3 \times 10^{-19} \mathrm{~W} \mathrm{~Hz}^{-1 / 2}$ and $3 \times 10^{-20} \mathrm{~W} \mathrm{~Hz}^{-1 / 2}$, respectively. ${ }^{1}$

*Address all correspondence to Steven Hailey-Dunsheath, haileyds@caltech.edu 
Table 1 Origins far-IR detector array targets and SPACEKIDs demonstrated performance.

\begin{tabular}{lccc}
\hline \hline Parameter & Origins-FIP target & Origins-OSS target & SPACEKIDs demo \\
\hline$\lambda(\mu \mathrm{m})$ & 50 to 250 (in two bands) & 25 to 588 (in six bands) & 350 \\
NEP $\left(\mathrm{W} \mathrm{Hz}^{-1 / 2}\right)$ & $3 \times 10^{-19}$ & $3 \times 10^{-20}$ & $3 \times 10^{-19 a}$ \\
Tile size (pixels) & 1 kilopixel + mosaicking to $\sim 10^{4}$ & 961 \\
Multiplexing & 2000 pixels per circuit, $4-\mathrm{GHz}$ BW available & 961 from 3.9 to $5.6 \mathrm{GHz}$ \\
Minimum pitch $(\mathrm{mm})$ & 0.5 & $0.4 \times 0.7$ & 1.6 \\
$\tau_{\text {det }}(\mathrm{ms})$ & $<3$ & $<3$ & 1.5 \\
Min. yield & $70 \%$ & $70 \%$ & $83 \%$ \\
Crosstalk $(\mathrm{dB})$ & $<-17$ & $<-17$ & $<-30$ \\
$1 / f$ knee $(\mathrm{Hz})$ & $<0.1$ & $<0.1$ & $<0.05^{\mathrm{a}}$ \\
Cosmic ray dead time & $<10 \%$ & $<10 \%$ & $<5 \%$ \\
\hline \hline
\end{tabular}

${ }^{\mathrm{a}}$ For the SPACEKIDs detectors, the frequency NEP is $3 \times 10^{-19} \mathrm{~W} \mathrm{~Hz}^{-1 / 2}$ at $60 \mathrm{Ho} 80 \mathrm{~Hz}$ audio frequency, but is dominated by $1 / f$ noise; the dissipation NEP is $6 \times 10^{-19} \mathrm{~W} \mathrm{~Hz}^{-1 / 2}$ with $\mathrm{a}<0.05 \mathrm{~Hz}$ knee and is limited by readout electronics. ${ }^{2}$

These values render the detector noise negligible compared with the photon noise from the solarsystem and galactic dust that ultimately limits far-IR measurements. The spectroscopy NEP target, in particular, has no application outside of a cryogenic space telescope, so improvement over the state-of-the-art is required; initial steps are underway and results are very promising. In addition, the array formats for both the imager and spectrometer are larger than anything flown thus far, though tractable when scaled from ground-based and suborbital experiments. The Origins far-IR detector needs are summarized in Table 1, and additional details can be found in the Origins 2019 Technology Development Plan. ${ }^{3}$

A number of superconducting detector technologies are under consideration for the far-IR instruments on Origins, including transition-edge-sensed (TES) bolometers (Sadlier et al., this volume), kinetic inductance detectors (KIDs), and quantum capacitance detectors (Echternach et al, this volume). Here, we discuss the prospects of KIDs to meet the requirements shown in Table 1.

\section{KIDs: A Brief Introduction}

The technology for semiconductor infrared array detectors is well developed, with silicon impurity band conduction detectors offering excellent performance in $1 \mathrm{k} \times 1 \mathrm{k}$ formats out to $\lambda=28 \mu \mathrm{m}$. ${ }^{4}$ Stressed gallium-doped germanium photoconductors have been used out to $\lambda=200 \mu \mathrm{m}$, e.g., on Spitzer/MIPS ${ }^{5}$ and Herschel/PACS $;{ }^{6}$ however, the difficulty of scaling this technology and the need for detectors beyond $200 \mu \mathrm{m}$ has driven strong interest in superconducting detectors. ${ }^{7-9}$ At present, two superconducting detector technologies have been demonstrated in large array formats at millimeter through far-IR wavelengths: TES bolometers ${ }^{10-12}$ and KIDs. ${ }^{13,14}$

In contrast to TES bolometers, which operate at the superconducting transition temperature $T_{c}$, KIDs operate at temperatures well below $T_{c}$. As a result, almost all of the conduction electrons in a KID are in the form of Cooper pairs. Unlike electrons in a normal metal, the Cooper pairs do not suffer scattering and can therefore carry current with no dissipation, leading to the hallmark property of superconductivity, the vanishing of the DC resistance. Indeed, the DC resistance of a KID is identically zero; a KID functions by sensing the (nonzero) AC impedance of the superconductor ${ }^{15}$ - the "kinetic inductance" - at RF or microwave frequencies. Meanwhile, a TES bolometer operates by sensing the temperature-dependent DC resistance 
at the onset of superconductivity, where the resistance is an appreciable fraction of its normalstate value.

Superconductivity has an energy scale and therefore a limited frequency range. The pairs have a binding or "gap" energy of $E_{g}=2 \Delta \approx 3.5 k_{B} T_{c}$, corresponding to a photon frequency of $\nu_{g}=2 \Delta / h \approx 75 \mathrm{GHz} \times\left(T_{c} / 1 \mathrm{~K}\right)$. This "gap frequency" is the minimum energy required for a photon to break a Cooper pair. At frequencies below $\nu_{g}$, a single photon cannot break a Cooper pair, so the material cannot efficiently absorb energy and thus behaves as a superconductor. However, for frequencies above $\nu_{g}$ the material can readily absorb energy and behaves as an ordinary (dissipative) normal metal.

KIDs are microwave resonators that use both of the behaviors described above to detect optical radiation. One approach is to construct the resonator using a lumped-element circuit (the lumped element KID; LEKID), ${ }^{16}$ as shown in Fig. 1. As illustrated in Fig. 1(a), a simple meandered thin-film superconducting trace can serve as an efficient radiation absorber for frequencies above the gap, $\nu_{g}$, provided the meander spacing, area filling factor, and normal-state sheet resistance of the superconductor are chosen appropriately. Simultaneously, the meander behaves as a low-loss superconducting inductor for frequencies below the gap, $\nu_{g}$. For an ordinary inductor, the stored energy is given by $L I^{2} / 2$, where $I$ is the current. For a KID, the inductance $L$ has two

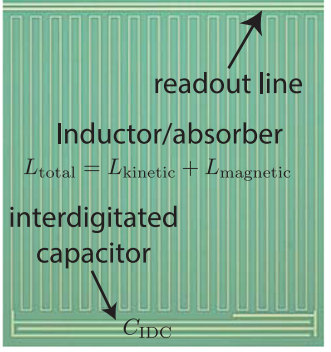

(a)

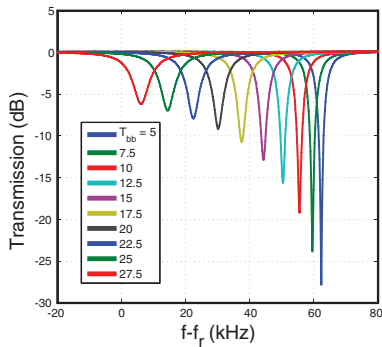

(b)

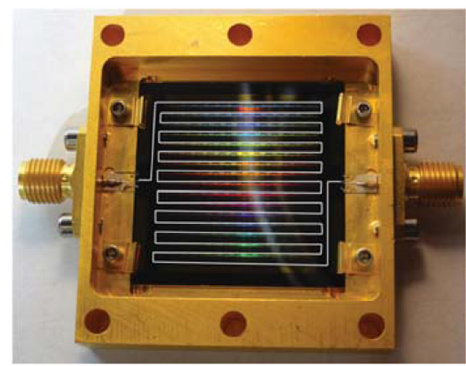

(c)

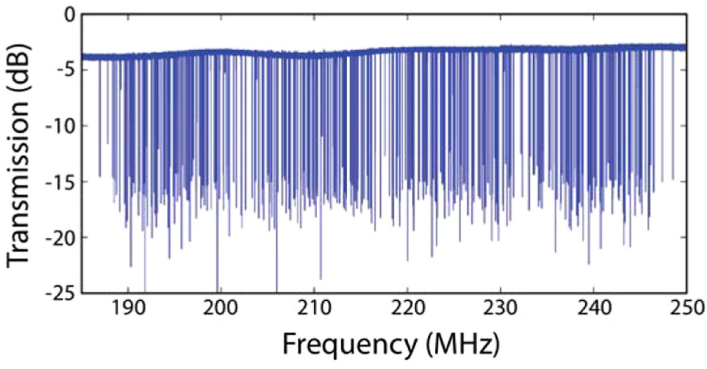

(d)

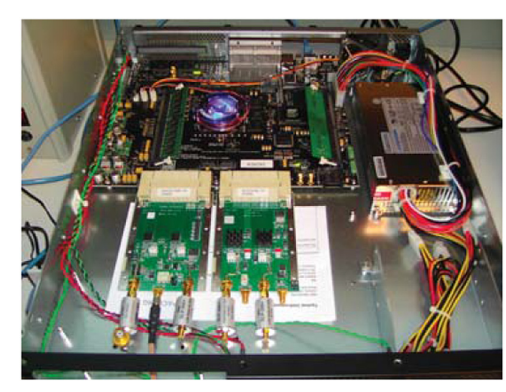

(e)

Fig. 1 (a) Close-up of a simple LEKID consisting of a meandered inductor $(L)$ that doubles as a
radiation absorber, and an interdigitated capacitor $\left(C_{\mathrm{IDC}}\right)$. These form an $L C$ resonant circuit that is weakly coupled to a common readout line. Reprinted with permission from Ref. 17. (b) The $1-\mathrm{GHz}$ resonant frequency of the KID shifts downward in response to the intensity of the radiation. These curves show measured data for a KID illuminated with blackbody radiation passed through a $\lambda=200 \mu \mathrm{m}$ bandpass filter. The intensity is controlled by the blackbody temperature, which was varied from $5 \mathrm{~K}$ to $27.5 \mathrm{~K}$. Note that a $10-\mathrm{kHz}$ shift represents a fractional change of $10 \mathrm{kHz} / 1 \mathrm{GHz}=10^{-5}$. Credit: P. K. Day. (c) A 432-pixel, $\lambda=350 \mu \mathrm{m} \mathrm{KID} \mathrm{array,} \mathrm{an} \mathrm{early} \mathrm{proto-}$ type for the MAKO instrument. ${ }^{18,19}$ The array is read out using frequency multiplexing. Readout signals are injected and extracted using two coaxial connectors; the readout line is highlighted in white. Credit: C. McKenney and H. G. Leduc. (d) A frequency sweep of the MAKO prototype array reveals 415 of 432 resonances with $>6 \mathrm{~dB}$ depth, corresponding to a fabrication yield of $96 \%$. Credit: C. McKenney. (e) Example of first-generation (ROACH-1) KID readout electronics. ${ }^{20}$ The circuit boards in the foreground contain fast digital-to-analog converters that generate the multifrequency analog signal that excites the KID array, and the analog-to-digital converters that digitize the return signal from the KID array. The FPGA, illuminated and underneath the cooling fan in the background, performs the digital signal processing needed to separate the individual KID frequencies. 


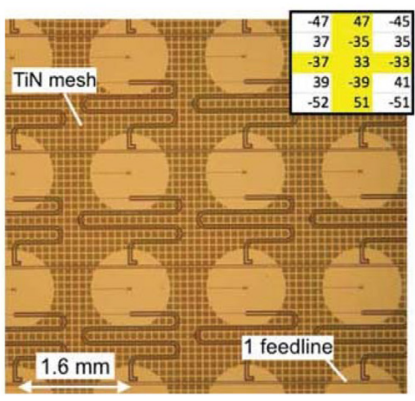

(a)

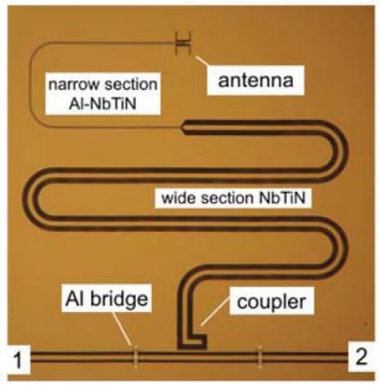

(b)

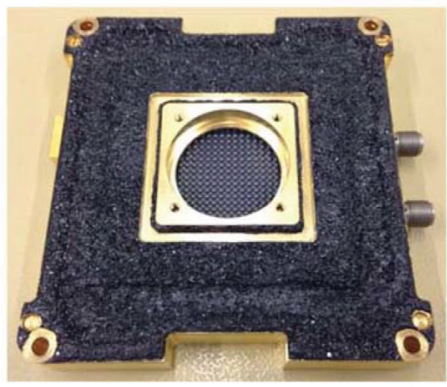

(c)

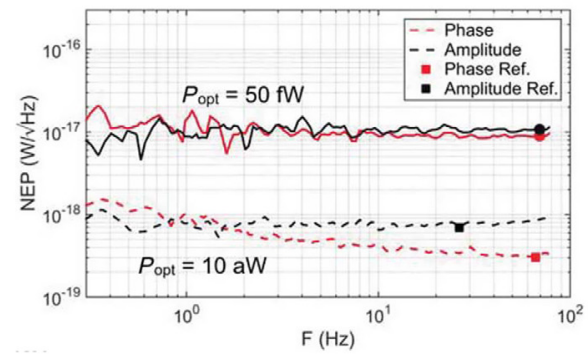

Fig. 2 SPACEKIDs detector figures from Ref. 2. Top: Photographs of the 961 pixel demonstration array highlighting (a) the TiN absorbing mesh located between the detector wafer and lens array, (b) the quarter-wave CPW hybrid NbTiN-AI KID and the coupled twin-slot antenna, and (c) the fully assembled detector package. Bottom: Measured NEP of a representative detector for phase and amplitude readout. Credit: Ref. 2, reproduced with permission ( ESO.

components: the ordinary magnetic inductance $L_{\text {magnetic }}$ corresponding to energy in the magnetic field and the kinetic inductance $L_{\text {kinetic }}$ corresponding to the kinetic energy of the Cooper pairs carrying the current. Like the energy in the magnetic field, the kinetic energy represents stored energy that can be recovered because the pairs do not scatter and the kinetic energy is not dissipated. By combining the meandered inductor with a simple interdigitated capacitor, an $L C$ circuit is formed whose resonant frequency is given by $\nu_{r}=1 /(2 \pi \sqrt{L C})$. The resonance can be very sharp (high $Q$ ) because the inductor is superconducting and has very low loss; the resonance frequency, typically chosen to be a few $\mathrm{GHz}$ or lower, is far below the gap frequency, $\nu_{r} \ll \nu_{g}$.

An alternative to the LEKID is the transmission-line resonator. ${ }^{13}$ One common implementation is the quarter-wave coplanar waveguide (CPW) design, an example of which is shown in Fig. 2. The inductance and capacitance per unit length of the CPW transmission line are $L^{\prime}$ and $C^{\prime}$, respectively. The resonance frequency is then set by the length of the segment, $l$, as $\nu_{r}=1 /\left(4 l \sqrt{L^{\prime} C^{\prime}}\right)$. Unlike a LEKID, the CPW resonator does not directly absorb optical radiation but uses an antenna to deposit optical photons at the shorted end of the resonator. An advantage of this optical coupling scheme is that the antenna and detector are decoupled and can therefore be optimized individually. However, designs that require a transmission line to couple the radiation from the antenna to the KID become unacceptably lossy at optical frequencies above the gap frequency (e.g., $\nu_{g}=1.1 \mathrm{THz}$ for NbTiN). A design that directly couples the antenna to the KID has been shown to extend the optical frequency range up to $2.8 \mathrm{THz}$, at the expense of an increased antenna complexity and tighter fabrication tolerances. ${ }^{21}$ The typical dimensions of CPW resonators result in the CPW KIDs being larger and operating at higher readout frequency than LEKIDs.

Because the kinetic inductance derives from the motion of Cooper pairs, it is sensitive to the pair density. Meanwhile, absorption of above-gap radiation causes the breaking of Cooper pairs, which reduces their density and increases the kinetic inductance. In turn, the increased kinetic inductance causes the resonance frequency to shift downward. This is the fundamental detection mechanism for KIDs and is illustrated using experimental data in Fig. 1. In addition, the breaking of pairs by the absorbed radiation also increases the density of single electrons, which do scatter, 
increasing the $(\mathrm{AC})$ resistive dissipation. This broadens the resonances and reduces their depth, as evident in Fig. 1. Either of these two responses - the shift in the frequency $\nu_{r}$ or the dissipation $Q_{r}^{-1}$ of the resonance-may be used for KID readout. This is the origin of terms such as "frequency readout" or "dissipation NEP" used in this paper and in the literature; the terms "phase readout" and "amplitude NEP" are used synonymously.

The narrow resonance produced by a single KID leads to the simple scheme for frequencydomain multiplexing shown in Fig. 1. For LEKIDs, the capacitor geometry is adjusted so each KID has a unique resonance frequency; for CPW KIDs, this is achieved by adjusting the length of each resonator. The readout electronics generate a superposition of frequencies to excite the array and then separate these frequencies in the output signal from the array to isolate the response of individual pixels. This task is now tractable as a result of advances in electronics, especially digital signal processing using field programmable gate arrays (FGPAs). Figure 1(e) shows an early example of KID readout electronics.

The deployments of DemoCam, ${ }^{22,23}$ MUSIC, ${ }^{24-26}$ and $\mathrm{MAKO}^{18}$ (Fig. 1) at the Caltech Submillimeter Observatory, as well as NIKA ${ }^{27}$ on the IRAM 30-m telescope, represent early demonstrations of prototype KID arrays. Since then, KIDs have enabled a number of large (multi-kilopixel) ground-based millimeter-wave and submillimeter-wave cameras, including NIKA2, ${ }^{28-30}$ and A-MKID on the APEX 12-m telescope,,${ }^{31,32}$ with TolTEC ${ }^{33,34}$ soon to be deployed on the 50-m Large Millimeter Telescope (LMT) in Mexico. A new 850- $\mu \mathrm{m}$ camera employing TolTEC-style KIDs is under development for the EAO/JCMT 15-m telescope on Mauna Kea, ${ }^{35,36}$ to replace the SCUBA-2 TES camera ${ }^{37}$ in several years. Intensity mapping ${ }^{38}$ using the [C II] $158 \mu \mathrm{m}$ fine structure line (redshifted to 200 to $300 \mathrm{GHz}$ at $z=5-8$ ) is a major goal for KID-based millimeter-wave, ground-based imaging spectrometers under development, including SuperSpec, ${ }^{39}$ DESHIMA, ${ }^{40}$ and CONCERTO. ${ }^{41}$ Balloon payloads using KIDs are being developed, e.g., for the Terahertz Intensity Mapper (TIM) ${ }^{42}$ and EXCLAIM, ${ }^{43}$ and have now been flown on OLIMPO ${ }^{44}$ and BLAST-TNG ${ }^{45}$ KID-based millimeter-wave cameras are now also being developed for other applications, e.g., security ${ }^{46}$ and maritime surveillance. ${ }^{47}$

The NEP requirements for Origins are more demanding than for any existing instruments and necessitate dedicated detector development. A European consortium has executed a EU Seventh Framework Programme (FP7) project, named SPACEKIDs, explicitly targeting the very low NEP for space astrophysics missions. The SPACEKIDs demonstration meets the sensitivity requirement of the FIP instrument (Table 1) and achieves a high level of system maturity. ${ }^{2}$ A separate effort is underway to develop KIDs for Origins based on an extension of the TIM detector design. ${ }^{48}$ The prospects of meeting the Origins detector goals with these two approaches are discussed below.

\section{Low NEP Demonstration of the SPACEKIDs Array}

The KID array that most closely meets the Origins requirements is the array developed in the EU-funded SPACEKIDs program, ${ }^{2}$ a 5 -year multinational research activity specifically targeting the needs of future far-IR space missions in astrophysics and earth science. The demonstration array is a $31 \times 31(961)$ pixel detector array optimized for imaging at $850 \mathrm{GHz}$ and is coupled to a readout electronics system ${ }^{49}$ developed under the SPACEKIDs program.

The basic pixel is an antenna-coupled hybrid NbTiN-Al device implemented as a quarterwave CPW resonator, shown in detail in Fig. 2. The hybrid design combines the low noise properties of a NbTiN resonator with the excellent responsivity of aluminum, which has been shown to produce quasiparticle lifetimes $>1.5 \mathrm{~ms} .{ }^{50,51}$ The $\mathrm{CPW}$ resonator is $\sim 6.3 \mathrm{~mm}$ long, producing a resonant frequency $f_{r} \sim 4.7 \mathrm{GHz}$. For $\sim 75 \%$ of its length the resonator is fabricated from 500-nm thick NbTiN with large features (the linewidth and slotwidth are both $20 \mu \mathrm{m}$ ), where both the choice of $\mathrm{NbTiN}^{52}$ and the large features are designed to minimize noise from two-level system (TLS) fluctuators on the surfaces of the capacitive portions of the device. ${ }^{53,54}$ Due to the high $T_{c}\left(T_{c}=15 \mathrm{~K}\right)$, the NbTiN film provides only a reactive load to optical frequencies $<1.1 \mathrm{THz}$. For the rest of the length, the central conductor is a narrow ( $2 \mu \mathrm{m}$ linewidth) section of aluminum, which is expected to absorb $>95 \%$ of the optical power provided by a twin-slot antenna. The antenna is fed by an antireflection-coated silicon microlens, and the end-to-end single-polarization optical efficiency is estimated to be $74 \%$. 
All 961 resonators in the array are capacitively coupled to a single CPW readout line at the open end of the resonator. The SPACEKIDs readout system is capable of reading up to 4000 pixels in a $2-\mathrm{GHz}$ bandwidth centered anywhere between 4.5 and $7.5 \mathrm{GHz}{ }^{49}$ Crosstalk levels (both optical and electrical) $<-30 \mathrm{~dB}$ are achieved by implementing aluminum bridges over the CPW readout line, intelligent positioning of neighboring resonators in frequency space, and a substoichiometric TiN $\left(T_{c}=0.6 \mathrm{~K}\right)$ absorbing mesh on the backside of the sapphire wafer. The latter also assists in the suppression of cosmic ray effects, ${ }^{55}$ by absorbing the athermal phonons generated by cosmic ray events in the substrate.

The NEP of a typical KID in the array is shown in Fig. 2. At low temperature (120 mK) and under low optical load (10 aW), the frequency noise is dominated by TLS noise. The noise PSD has a nonwhite spectrum, and at high audio frequency (60 to $80 \mathrm{~Hz}$ ), the NEP is $3 \times 10^{-19} \mathrm{~W} \mathrm{~Hz}^{-1 / 2}$. In the amplitude quadrature, the white NEP is $6 \times 10^{-19} \mathrm{~W} \mathrm{~Hz}^{-1 / 2}$ and is dominated by a combination of the cold and warm readout electronics. The $1 / f$ knee in the amplitude noise PSD is $<0.05 \mathrm{~Hz}$.

The NEP of these detectors could be further reduced through an increase in the responsivity or a decrease in the electrical noise. Increasing the responsivity through a longer quasiparticle lifetime is not a practical solution, given the $\left(\tau_{\mathrm{det}}<3 \mathrm{~ms}\right)$ requirement (Table 1$)$. A more plausible path forward ${ }^{50}$ would be to decrease the $V_{L} \approx 130 \mu \mathrm{m}^{3}$ active volume of the detector. Reductions to the TLS noise could potentially be achieved by optimizing the capacitor geometry and choice of substrate material ${ }^{52}$ and/or the removal of exposed substrate surfaces in high-field regions ${ }^{56}$ Reducing the dissipation NEP could be achieved by optimizing the coupling to the readout line, which increases the KID amplitude noise with respect to the readout noise. Reference 2 suggested such changes could reduce the NEP down to a few $10^{-19} \mathrm{~W} \mathrm{~Hz}^{-1 / 2}$ down to $0.1 \mathrm{~Hz}$, but further reduction would demand more significant measures.

In Table 1, we compare the performance of the SPACEKIDs system with some of the key goals for the Origins detectors. The SPACEKIDs system achieves the desired NEP for the FIP instrument, as well as many other key requirements including the achieved tile size, multiplexing density, and cosmic ray dead time.

\section{Low NEP Lumped Element KIDs}

Direct-absorbing lumped element KIDs are promising candidates for the far-IR instruments on Origins. As described below, a model for an Origins appropriate detector leverages the demonstration of a feedhorn-coupled, low-volume aluminum device at $350 \mu \mathrm{m}$. Further reductions to the inductor volume may be implemented to reduce the NEP below $10^{-19} \mathrm{~W} \mathrm{~Hz}^{-1 / 2}$, and modifications to the absorber will allow operation over the full far-IR band.

\subsection{Aluminum LEKIDs for TIM}

TIM is a balloon-borne spectrometer that will operate at 240 to $420 \mu \mathrm{m}$, with a required detector NEP of $1 \times 10^{-17} \mathrm{~W} \mathrm{~Hz}^{-1 / 2}{ }^{42}$ The detectors will be horn-coupled LEKIDs, shown in Fig. 3, and described more fully in Ref. 48.

The absorber cross-section is limited to $300 \mu \mathrm{m}$ in diameter to minimize the detector volume, whereas the large capacitor occupies $\sim 50 \%$ of the pixel area to decrease the capacitor (TLS) noise. A conical feedhorn coupled to a circular waveguide is used to concentrate light on the absorber, maintaining a high filling factor, $2.3-\mathrm{mm}$ pitch hexagonal-packed focal plane. The inductor/absorber is a single meander of $0.4-\mu \mathrm{m}$ wide aluminum, patterned to provide an optimal impedance match to the horn's output waveguide. Segments of meander line are placed in close proximity at the corners, creating capacitive shorts at the optical frequencies that cause the meander to behave as a square mesh and thereby couple efficiently to both polarizations. This is achieved with a $0.3-\mu \mathrm{m}$ gap and a $0.6-\mu \mathrm{m}$ overlap length for each of the corners. The pixel is fabricated on a silicon on insulator wafer and has a $27-\mu \mathrm{m}$ thick backshort, created by etching from the backside to a buried oxide layer, then depositing gold. Electromagnetic simulations indicate band-averaged (790 to $900 \mathrm{GHz}$ ) coupling efficiencies of $90 \% .^{57}$ The 


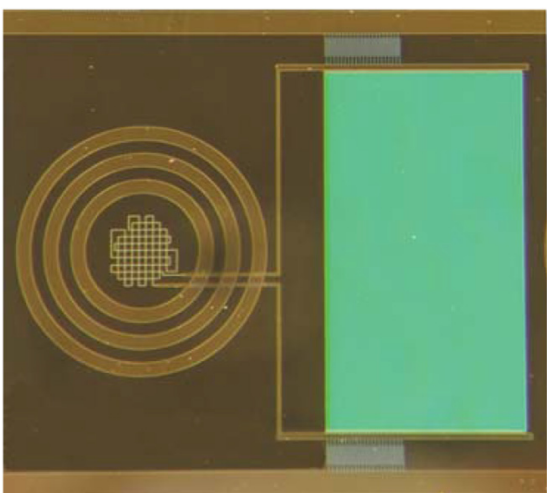

(a)

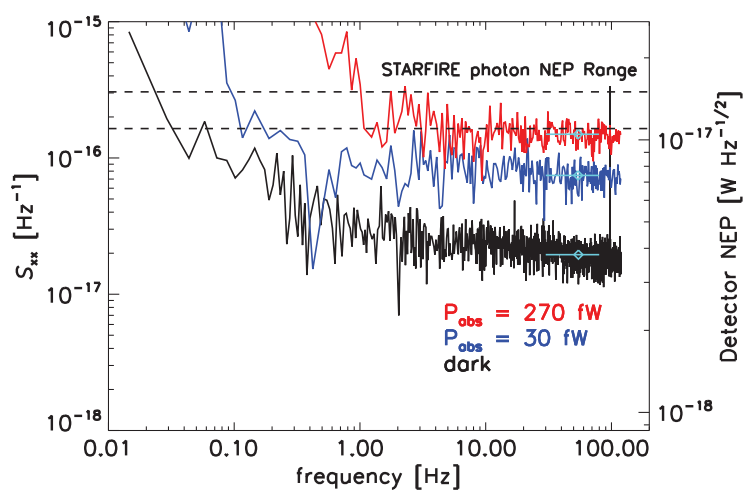

(b)

Fig. 3 TIM prototype detector. (a) Microscope image of a single pixel within a 45-pixel prototype array. The meandered inductor is surrounded by a set of optical choke rings and connected to a large interdigitated capacitor to form a 250-MHz LEKID. The inductor/absorber is illuminated by a conical feedhorn coupled to a circular waveguide. (b) Amplifier-subtracted $S_{x x}$ for a representative detector measured under various optical loads at $350 \mu \mathrm{m}$. At low loading the white noise corresponds to a detector NEP of $4 \times 10^{-18} \mathrm{~W} \mathrm{~Hz}^{-1 / 2}$. Figure reprinted with permission from the Journal of Low Temperature Physics.

interdigitated capacitor has $1-\mu \mathrm{m}$ wide fingers with $2-\mu \mathrm{m}$ gaps. When patterned from a $40-\mathrm{nm}$ film, the total inductor volume is $76 \mu \mathrm{m}^{3}$ and the resonant frequency is $\approx 250 \mathrm{MHz}$.

Initial tests were conducted with a 45-pixel prototype array of TIM detectors patterned on a standard silicon wafer, excluding the backshort. ${ }^{48}$ Figure 3 shows the noise spectrum for a typical KID measured dark and with optical loading, at a $210-\mathrm{mK}$ base temperature. At $30 \mathrm{fW}$ of optical loading the detector NEP is $4 \times 10^{-18} \mathrm{~W} \mathrm{~Hz}^{-1 / 2}$, with a $1 / f$ knee at $\approx 0.5 \mathrm{~Hz}$.

\subsection{Ultralow NEP Far-IR LEKIDs}

The TIM detector design presented in Sec. 4.1 may be modified to produce an increased responsivity and reduced NEP through a combination of a lower operating temperature, increased $\tau_{\mathrm{qp}}$ and reduced inductor volume.

\subsubsection{Increasing the responsivity}

The fractional frequency responsivity of a $\mathrm{KID}, R_{x}$, is given as

$$
R_{x}=\frac{\alpha \gamma S_{2}(\omega)}{4 N_{0} \Delta_{0}} \frac{\eta_{o} \tau_{q p}}{\Delta_{0} V_{L}}
$$

where $\alpha$ is the kinetic inductance fraction, $\gamma=1$ for thin films, $\eta_{o}$ is the pair-breaking efficiency, $N_{0}$ is the density of states, $\Delta_{0}$ is the gap energy, $V_{L}$ is the inductor volume, and $S_{2}(\omega)$ relates changes in the kinetic inductance to changes in the quasiparticle number density. ${ }^{14}$ For a superconductor in thermal equilibrium, the $\left(n_{\mathrm{qp}} \tau_{\mathrm{qp}}\right)$ product is a material-specific constant

$$
n_{\mathrm{qp}} \tau_{\mathrm{qp}}=\frac{N_{0} \tau_{0}\left(k_{B} T_{c}\right)^{3}}{2 \Delta^{2}}
$$

where $\tau_{0}$ is the characteristic electron-phonon interaction time. ${ }^{58}$ The quasiparticle lifetime has been observed to saturate at a maximum value $\tau_{\max }$ in the low temperature limit of some dark resonators, ${ }^{59,60}$ leading to a commonly applied empirical parameterization

$$
\tau_{\mathrm{qp}}=\frac{\tau_{\max }}{1+n_{\mathrm{qp}} / n^{*}},
$$




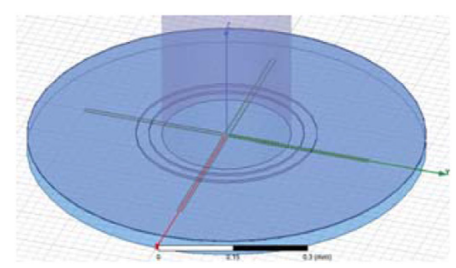

(a)

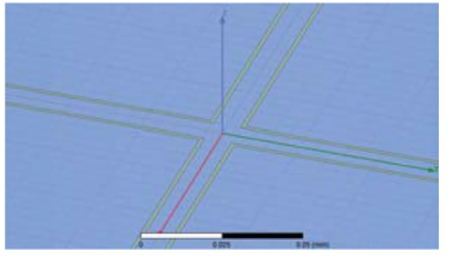

(b)

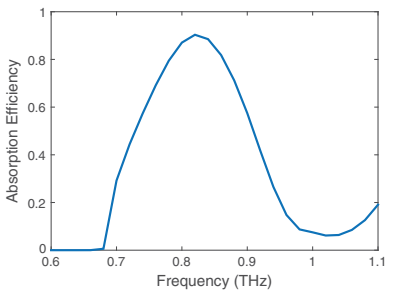

(c)

Fig. 4 (a) Geometry for a dual-polarization, minimal volume aluminum absorber coupled to a circular waveguide. (b) Zoom-in of the center of the absorber, showing the metal film (green) on the silicon substrate (blue). The linewidth is $150 \mathrm{~nm}$ and the total volume is $10 \mu \mathrm{m}^{3}$ for a $20-\mathrm{nm}$ film thickness. (c) HFSS-simulated absorption efficiency, indicating a peak of $90 \%$ at $360 \mu \mathrm{m}$. Credit: P. K. Day.

for some critical $n^{*}$ value. Consistency between Eqs. (2) and (3) in the high $n_{\mathrm{qp}}$ limit requires

$$
n^{*} \tau_{\max }=\frac{N_{0} \tau_{0}\left(k_{B} T_{c}\right)^{3}}{2 \Delta^{2}}
$$

The $\tau_{\mathrm{qp}}$ inferred from the noise measurements of the TIM devices is consistent with Eqs. (3) and (4) with $\tau_{\max }=35 \mu \mathrm{s}$ and $\tau_{0}=139 \mathrm{~ns}$. This $\tau_{\max }$ value is unexpectedly short; $\tau_{\mathrm{qp}} \gtrsim 500 \mu \mathrm{s}$ is commonly achieved in aluminum resonators operated below $150 \mathrm{mK}$ in dark conditions. ${ }^{50,59,61,62}$ While the shorter $\tau_{\mathrm{qp}}$ achieved in the TIM devices may be in part due to the higher operating temperature, disorder in the material is potentially a more important factor, ${ }^{60}$ this may be addressed by optimizing the fabrication process. Quarter-wavelength CPW devices recently fabricated at JPL yielded quasiparticle lifetimes of 1 to $5 \mathrm{~ms}$ for film thicknesses of 20 to $50 \mathrm{~nm} .^{63}$ We are therefore optimistic the fabrication may be optimized to achieve $\tau_{\max }=1 \mathrm{~ms}$. Achieving an aluminum film with $\tau_{\max }=1 \mathrm{~ms}$ and $\tau_{0}=139 \mathrm{~ns}$ would lead to $\tau_{\mathrm{qp}}=992 \mu \mathrm{s}$ in a dark device at $150 \mathrm{mK}$.

The responsivity may be further increased by reducing the inductor/absorber volume. The geometry currently used provides an impedance match to the incident radiation over the full waveguide cross-section. However, a single linear structure cutting across the center of the circular footprint has been shown to couple well to the fundamental waveguide mode ${ }^{64}$ and has a much smaller volume. We have designed a dual-polarization sensitive absorber that achieves a simulated peak absorption efficiency of $90 \%$ and reduces the inductor volume from 76 to $10 \mu \mathrm{m}^{3}$ for a 20-nm film thickness (Fig. 4). This absorber uses a 150-nm linewidth, which pushes the limits of UV lithography but may be easily achieved with electron-beam lithography. Due to the narrower linewidth, the inductance of this lower volume absorber is close to that in the current TIM device, so may be implemented while keeping the readout frequency constant at $\approx 250 \mathrm{MHz}$.

\subsubsection{Noise model}

The dark noise measurements of the TIM detectors indicate a limiting noise level of $S_{\mathrm{xx}, 0}=1.8 \times 10^{-17} \mathrm{~Hz}^{-1}$ (Fig. 3). While the source of this noise floor is not yet fully understood, we may adopt it as an upper limit to the TLS noise at $210 \mathrm{mK}$ and $10 \mathrm{~Hz}$ audio frequency. These noise measurements were obtained with a drive power of $P_{g} \approx-110 \mathrm{dBm}$, which is $\approx 5 \mathrm{~dB}$ below bifurcation. Under these conditions, the resonators are characterized by $Q_{i} \approx Q_{c} \approx 10^{5}$.

For a fixed capacitor geometry, the TLS noise has a well-characterized dependence on operating temperature and internal power: $S_{\mathrm{xx}, \mathrm{TLS}} \propto P_{\text {int }}^{-1 / 2} T^{-1.7},{ }^{65}$ where $P_{\text {int }}=\left(2 Q_{r}^{2} P_{g}\right) /\left(\pi Q_{c}\right)$. As we consider changing the drive power and temperature in our proposed low volume KIDs, we therefore assume the TLS noise will be 


$$
S_{\mathrm{xx}, \mathrm{TLS}}=1.8 \times 10^{-17} \mathrm{~Hz}^{-1}\left(\frac{4 Q_{r}^{2}}{10^{5} Q_{c}}\right)^{-1 / 2}\left(\frac{P_{g}}{10^{-14} \mathrm{~W}}\right)^{-1 / 2}\left(\frac{T}{210 \mathrm{mK}}\right)^{-1.7} .
$$

The bifurcation power will be reduced with the smaller volume. ${ }^{66}$ Additionally specifying that we maintain the drive power at least $5 \mathrm{~dB}$ below bifurcation leads to the constraint

$$
P_{g} \leq 10^{-14} \mathrm{~W}\left(\frac{f_{r}}{250 \mathrm{MHz}}\right)\left(\frac{V_{L}}{76 \mu \mathrm{m}^{3}}\right)\left(\frac{Q_{c}}{10^{5}}\right)\left(\frac{10^{5}}{2 Q_{r}}\right)^{3} .
$$

The fractional frequency noise contributed by an LNA with noise temperature $T_{n}$ and zero detuning may be written as ${ }^{14}$

$$
S_{\mathrm{xx}, \mathrm{LNA}}=\frac{4}{\chi_{c}^{2} Q_{i}^{2}} \frac{k_{B} T_{n}}{P_{g}}
$$

with

$$
\chi_{c}=\frac{4 Q_{c} Q_{i}}{\left(Q_{c}+Q_{i}\right)^{2}}
$$

We assume $T_{n}=5 \mathrm{~K}^{67}$

Generation-recombination noise is given as ${ }^{14}$

$$
\mathrm{NEP}_{\mathrm{GR}}^{2}=2 h \nu P_{o}\left(1+n_{0}\right)+\frac{4 \eta_{a} \chi_{\mathrm{qp}} \Delta_{0}}{\eta_{o}^{2}} P_{a}+\frac{4 \Delta_{0}^{2}}{\eta_{0}^{2}}\left(\Gamma_{\mathrm{th}}+\Gamma_{r}\right),
$$

where $\eta_{0} \approx 0.57$ is the pair-breaking efficiency, $P_{a}$ is the absorbed readout power, $\eta_{a}$ is the efficiency with which absorbed readout power is converted to quasiparticles, $\chi_{\mathrm{qp}}=Q_{i} / Q_{\mathrm{qp}}$, and $\Gamma_{\mathrm{th}}$ and $\Gamma_{r}$ are the thermal generation and total quasiparticle recombination rates, respectively. The four terms in Eq. (9) represent photon generation noise, readout power generation noise, thermal generation noise, and recombination noise, respectively.

\subsubsection{NEP optimization}

Given the noise terms described in Sec. 4.2.2, our low volume KID may be optimized to achieve the minimum NEP for a given optical loading. Here, we consider this optimization assuming a $P_{o}=10^{-18} \mathrm{~W}$ load at $\lambda=350 \mu \mathrm{m}$, as appropriate for OSS. We assume $V_{L}=10 \mu \mathrm{m}^{3}$ and vary $T$ and $P_{g}$. We assume our devices maintain $Q_{i}=Q_{c}=10^{5}$.

A considerable uncertainty in this optimization is the pair-breaking efficiency of the readout power, $\eta_{a}$. The absorption of microwave photons will drive the quasiparticle distribution out of thermal equilibrium and increase the number density $n_{\mathrm{qp}}{ }^{50,68,69}$ These excess quasiparticles will reduce the responsivity, and this absorption will be the source of an additional shot noise term. Reference 50 measures $\eta_{a} \sim 10^{-3}$ for a $f_{r}=5.3 \mathrm{GHz}$ resonator with a similar readout power density (between $10^{-16}$ and $10^{-15} \mathrm{~W} \mu \mathrm{m}^{-3}$ ) as envisioned for our low volume devices, whereas Ref. 69 calculates $\eta_{a} \sim 0.1-0.5$ in simulations of a $f_{r}=4 \mathrm{GHz}$ resonator with absorbed power densities between $10^{-19}$ and $10^{-14} \mathrm{~W} \mu \mathrm{m}^{-3}$. This efficiency may be expected to depend on the resonator frequency and will likely be different given the lower frequency $\left(f_{r} \approx 250 \mathrm{MHz}\right)$ considered here. We choose to adopt $\eta_{a}=0.1$ in our modeling, near the upper end of the above estimates.

In Figs. 5 and 6, we show the contributions of the various noise terms as a function of $T$ and $V_{L}$. For the TLS noise value adopted here, an operating temperature of $150 \mathrm{mK}$ represents a good balance between rising TLS noise at lower temperatures and rising thermal GR noise at higher temperatures (Fig. 5). For $V_{L}=10 \mu \mathrm{m}^{3}$, the total NEP is minimized by maximizing the readout power, subject to the limit described in Eq. (6). The detector noise is $\mathrm{NEP}_{\text {det }}=$ $4 \times 10^{-20} \mathrm{~W} \mathrm{~Hz}^{-1 / 2}$ and is dominated by TLS noise. This model is summarized in Table 2 . 


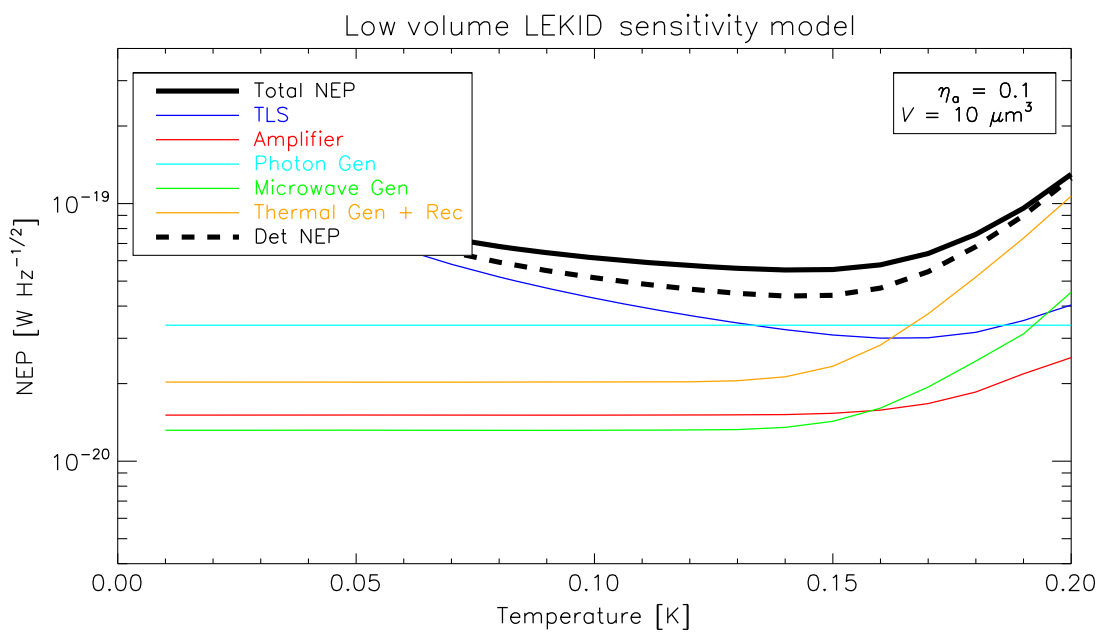

Fig. 5 Sensitivity model predictions for the low volume LEKID proposed here, with $\eta_{a}=0.1$, $V_{L}=10 \mu \mathrm{m}^{3}$, and maximized $P_{g}$ (see text for details). Detector NEP (black dashed) includes all contributions to the NEP aside from the photon generation noise. The optimal operating temperature is $T \approx 150 \mathrm{mK}$.

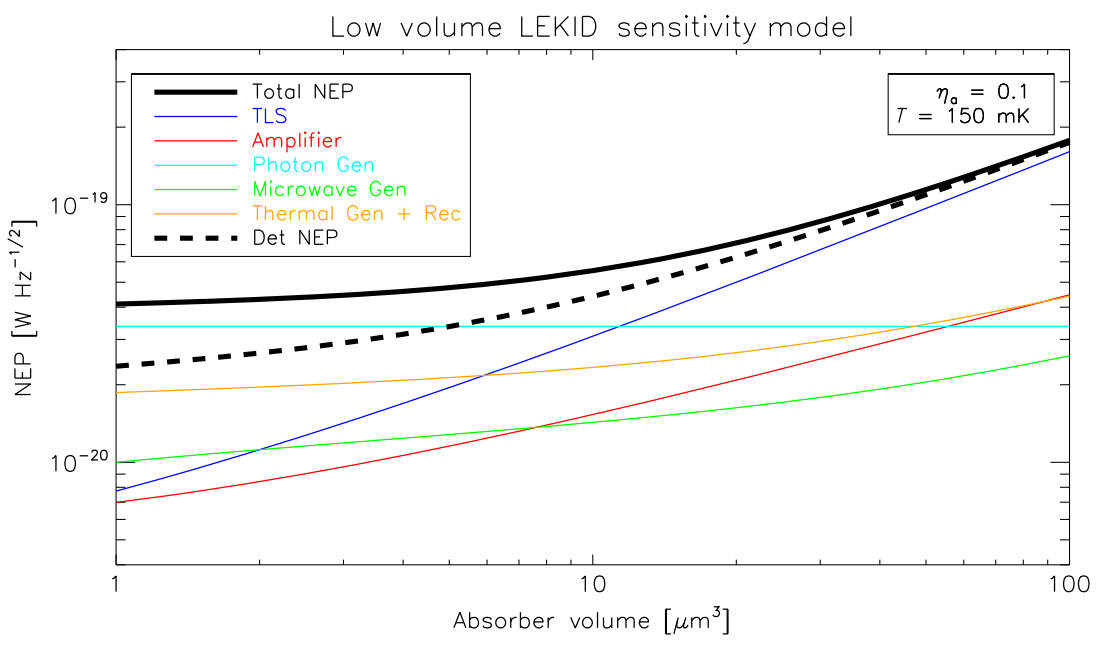

Fig. 6 Same as Fig. 5 with $T=150 \mathrm{mK}$. With an optical load of $P_{o}=10^{-18} \mathrm{~W}$, the photon generation noise is $\mathrm{NEP}_{\text {photon }}=3.4 \times 10^{-20} \mathrm{WHz}^{-1 / 2}$, and the detector noise with $V_{\mathrm{L}}=10 \mu \mathrm{m}^{3}$ is $\mathrm{NEP}_{\text {det }}=4.4 \times 10^{-20} \mathrm{~W} \mathrm{~Hz}^{-1 / 2}$. Further reductions in the detector NEP are possible by further reducing $V_{\mathrm{L}}$.

We note that further reductions to the inductor volume will reduce the NEP (Fig. 6). Such reductions are possible with the absorber shown in Fig. 4 by reducing the linewidth below $150 \mathrm{~nm}$.

\subsection{Ultralow NEP LEKIDs at $25 \mu \mathrm{m}$}

The TIM detectors described in Sec. 4.1 may be adapted to operate at shorter wavelengths with the use of a different absorber geometry. The wire grid absorber used in the $350 \mu \mathrm{m}$ detectors is $0.4 \mu \mathrm{m}$ wide; scaling this design with wavelength would require prohibitively narrow lines at $\lambda<100 \mu \mathrm{m}$. An alternative approach is to interrupt the absorber line with a meander that increases the resistance per unit length while simultaneously introducing a distributed capacitance to compensate for the accompanying distributed inductance. ${ }^{70}$ An impedance match to silicon may then be maintained with much wider lines. This effort is complementary to and also driven by the mid-IR KID development for the Galaxy Evolution Probe, which requires detectors down to $10 \mu \mathrm{m}$ wavelength. ${ }^{71}$ 
Table 2 Proposed low NEP designs.

\begin{tabular}{|c|c|c|c|c|}
\hline \multirow[b]{2}{*}{ Parameter } & \multicolumn{2}{|c|}{ Existing $350 \mu \mathrm{m}$} & \multicolumn{2}{|c|}{$\begin{array}{l}\text { Proposed low-NEP } \\
\text { lumped element }\end{array}$} \\
\hline & SPACEKIDs & TIM lumped element & $350 \mu \mathrm{m}$ & $25 \mu \mathrm{m}$ \\
\hline Temp (mK) & 120 & 210 & 150 & 150 \\
\hline$\tau_{\max }(\mu \mathrm{s})$ & 1480 & 35 & 1000 & 1000 \\
\hline Linewidth (nm) & 2000 & 400 & 150 & 200 \\
\hline$V_{L}\left(\mu \mathrm{m}^{3}\right)$ & 130 & 76 & 10 & 54 \\
\hline$f_{\text {res }}(\mathrm{MHz})$ & 4700 & 250 & 250 & 1700 \\
\hline Pitch (mm) & 1.6 & 2.3 & 2.3 & 0.4 \\
\hline$\alpha$ (kin. ind. fraction) & 0.09 & 0.76 & 0.76 & 0.76 \\
\hline$R_{x}\left(\mathrm{~W}^{-1}\right)$ & $4 \times 10^{9}$ & $1.2 \times 10^{9}$ & $3.1 \times 10^{11}$ & $5.4 \times 10^{10}$ \\
\hline$S_{\mathrm{xx}, \mathrm{TLS}}\left(\mathrm{Hz}^{-1}\right)$ & $1.4 \times 10^{-18}$ & $1.8 \times 10^{-17}$ & $9.0 \times 10^{-17}$ & $3.4 \times 10^{-16}$ \\
\hline $\mathrm{NEP}_{\text {det }}\left(\mathrm{WHz}^{-1 / 2}\right)$ & $3 \times 10^{-19}$ & $4 \times 10^{-18}$ & $4.4 \times 10^{-20}$ & $3.5 \times 10^{-19}$ \\
\hline Notes & 1 & 2 & 3 & 4 \\
\hline
\end{tabular}

Note: $350 \mu \mathrm{m}$ designs include (1) the SPACEKIDs device as shown in Fig. $2{ }^{2}{ }^{2}(2)$ the fabricated/characterized TIM detector, (3) the same TIM device with an increased $\tau_{\max }$, reduced temperature, and reduced inductor volume under a 1-aW optical load. (4) $25 \mu \mathrm{m}$ design adapts the low volume $350 \mu \mathrm{m}$ device to optically couple at shorter wavelengths, evaluated at 0.5 -aW load.

This concept is shown in Fig. 7. The absorber couples to vertically polarized radiation and is designed to be backside-illuminated through the silicon substrate. For a $10-\mu \mathrm{m}$ wavelength configuration, the linewidth is $200 \mathrm{~nm}$ and the unit cell is $2.4 \mu \mathrm{m}$ on a side. Agilent HFSS simulations indicate that this geometry achieves a peak single-polarization absorptivity of just over $70 \%$ near $10 \mu \mathrm{m}$, and performs well at wavelengths beyond $25 \mu \mathrm{m}$, the shortest wavelength required for Origins. This linewidth can be fabricated with a UV stepper, and further optimization targeting $25 \mu \mathrm{m}$ would allow even larger linewidths. In addition, Ref. 70 presented a resonant dual-polarization design for which simulations yield absorption of $70 \%$ in each polarization.

Coupling these absorbers to free space radiation is achieved with microlenses. Microlenses are commercially available at near-infrared wavelengths, but their substrates do not have low absorption at mid-infrared wavelengths. Thus, they should be made of silicon for wavelengths $>20 \mu \mathrm{m}$ (and likely germanium for 10 to $20 \mu \mathrm{m}$ because of the silicon absorption features in the 14 to $16 \mu \mathrm{m}$ range). Microlens arrays could either be fabricated separately and bonded to backilluminated KID arrays ${ }^{73}$ or etched into the backside before or after KID fabrication. There are numerous examples of silicon microlens fabrication in the literature that could achieve the $\mu \mathrm{m}$-level tolerances required for operation down to $25 \mu \mathrm{m},{ }^{74-80}$ although those involving micromachining and laser etching could be slow for arrays of $10^{4}$ detectors. Another promising approach is the deposition of micro-Fresnel plate lenses. ${ }^{81}$ This approach has the virtue of fabrication simplicity but rejects $50 \%$ of the radiation outright.

Reference 70 presented dark measurements of an array of KIDs with an absorber as shown in Fig. 7. The aluminum thickness was $40 \mathrm{~nm}$, resulting in an inductor volume of $54 \mu \mathrm{m}^{3}$. The IDCs were smaller than those used in the TIM detectors, reducing the pitch to $0.4 \mathrm{~mm}$ and pushing the readout frequencies up to the 1.4 to $2.0 \mathrm{GHz}$ range. The frequency noise was dominated by TLS noise, with typical values at $10 \mathrm{~Hz}$ of $S_{\mathrm{xx}}=(3-0.6) \times 10^{-16} \mathrm{~Hz}^{-1}$ at $T=100$ to $200 \mathrm{mK}$, consistent with the expected $T^{-1.7}$ scaling. Assuming the same film properties as in Sec. 4.2.1 allows an estimate of the NEP. For a 0.5-aW load (appropriate for OSS at $25 \mu \mathrm{m}$ ), 


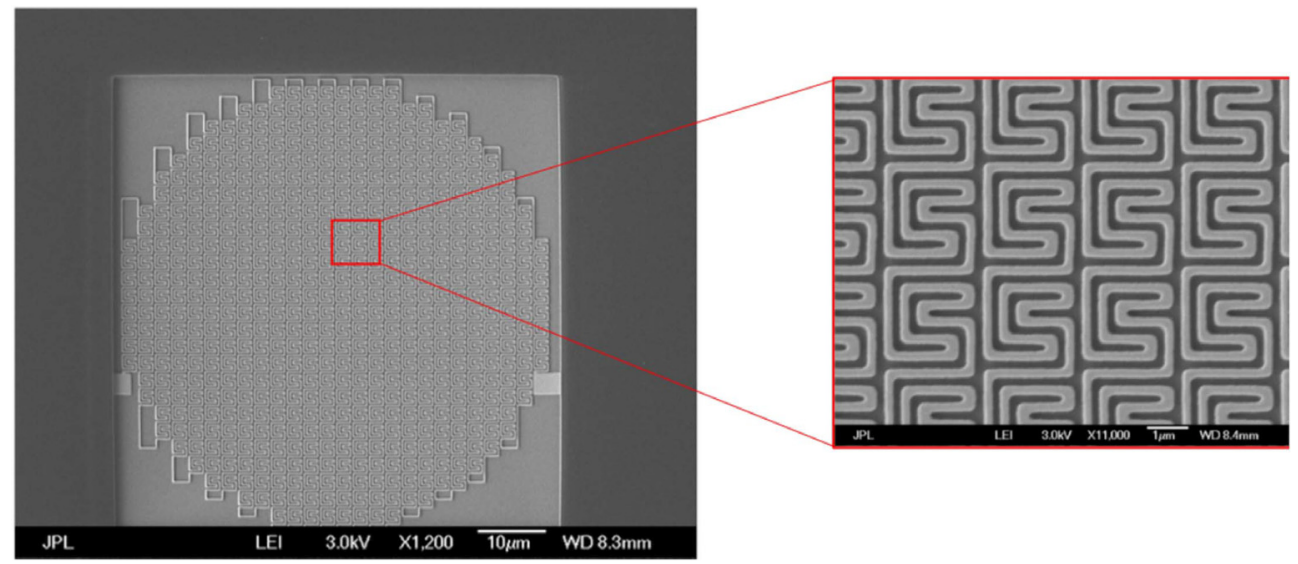

Fig. 7 Photograph of the inductor/absorber portion of a 10- $\mu \mathrm{m}$ LEKID. The inset shows the basic unit cell, optimized for the absorption of vertically polarized radiation, replicated over the full $60-\mu \mathrm{m}$ diameter absorber area. Simulations indicate a peak single-polarization absorption efficiency of $70 \%$ near $10 \mu \mathrm{m}$. Figure duplicated unaltered from Ref. 70; license can be found at Ref. 72 .

the predicted responsivity is $R_{x}=5.4 \times 10^{10} \mathrm{~W}^{-1}$, and the detector noise is strongly limited by TLS with an NEP of $4 \times 10^{-19} \mathrm{~W} \mathrm{~Hz}^{-1 / 2}$. These parameters are included in Table 2 .

In summary, lumped element KIDs appear to be viable for Origins at wavelengths as short as $25 \mu \mathrm{m}$. Simulations indicate good optical efficiency for microlens-coupled absorbers, and dark noise measurements indicate NEPs matching the FIP instrument goal.

\section{Detector Array Requirements}

In addition to the individual detector NEP goals, there are a number of array-level requirements for FIP and OSS summarized in Table 1. Many of these requirements (e.g., tile size and multiplexing density) are met with current laboratory and suborbital demonstrators, whereas others (e.g., the broad spectral range, small pixel pitch, and cosmic ray suppression) represent technical challenges.

\subsection{Array Size and Multiplexing Density}

The FIP and OSS instruments will each have of order $\sim 10^{5}$ detectors, in multiple arrays of up to 16,000 pixels each. The arrays will be tiled from $\sim 1$ to 4 kilopixel subarray dies. Kilopixel arrays have been fielded on ground-based telescopes ${ }^{18,29}$ and larger arrays are near deployment (e.g., the TolTEC millimeter-wave camera with three arrays, including one that is 3.6 kilopixels $^{34}$ ). The SPACEKIDs demonstration array has 961 pixels fabricated on a single wafer, and with stringent criteria on allowed crosstalk achieves a $>83 \%$ yield. $^{2}$

The digital readout electronics baselined for Origins adopt a multiplexing density of 2000 pixels in a 4-GHz band, or 500 detectors/GHz. The SPACEKIDs array is designed to place 961 channels in $1.7 \mathrm{GHz}$ of bandwidth, a density of about 565 pixels/GHz. The potential for crosstalk between adjacent channels in readout space is minimized by designing for a high $Q=10^{5}$ and scheduling the readout frequencies to keep nearest neighbors in readout space at least one detector apart while maintaining a smooth variation in readout frequency over the wafer to minimize scatter due to film variations. A small fraction of devices is found to be close enough in readout space to exhibit crosstalk at the 30-dB level.

Operating at lower $f_{r}$ offers a natural multiplexing advantage, as for a fixed $Q$ the bandwidth per resonator is correspondingly reduced. ${ }^{18}$ Additional improvements in multiplexing density are possible with LEKIDs using post-fabrication resonator trimming methods, which can adjust resonant frequencies after fabrication to eliminate frequency-space collisions arising from lithography errors and material variation. ${ }^{82,83}$ 


\subsection{Spectral Coverage and Pitch}

The FIP and OSS instruments will cover $\lambda=50$ to $250 \mu \mathrm{m}$ and $\lambda=25$ to $588 \mu \mathrm{m}$, respectively (Table 1). The SPACEKIDs detectors discussed in Sec. 3 are limited to operation at $\lambda>270 \mu \mathrm{m}$ due to the use of a NbTiN ground plane in the antenna. However, the use of thick aluminum as a ground plane allows operation at higher frequencies with only $10 \%$ additional loss. ${ }^{50}$ An extension of this design to higher frequencies and larger bandwidths has been demonstrated with the use of a leaky lens antenna, which couples to radiation over an octave of bandwidth from 107 to $215 \mu \mathrm{m} .{ }^{21}$ While the resonator design is complex, the KIDs used in this demonstration achieved a limiting $\mathrm{NEP} \approx 2.5 \times 10^{-19} \mathrm{~W} \mathrm{~Hz}^{-1 / 2}$. Extending this design down to $25 \mu \mathrm{m}$ would require fabricating $300 \mathrm{~nm}$ features and maintaining a $1-\mu \mathrm{m}$ air gap between the lens and antenna. As discussed in Sec. 4.3, the LEKIDs under development for $25 \mu \mathrm{m}$ operation use a lens-coupled absorber with a $200 \mathrm{~nm}$ or larger linewidth. A version with $200 \mathrm{~nm}$ features has been successfully patterned with good yield and characterized dark.

Because FIP and OSS span the full far-IR band, the minimum required pixel pitches are smaller than in the existing prototypes designed for $\lambda=350 \mu \mathrm{m}$. The pitch for FIP is $500 \mu \mathrm{m}$ in both bands, whereas for OSS it is $400 \times 700 \mu \mathrm{m}$ at the shortest wavelength. This is significantly smaller than the $1.6 \mathrm{~mm}$ size of the quarter-wave CPW resonator used for the SPACEKIDs demonstrator and requires reducing the per-pixel area by a factor of $\sim 10$. The $10-\mu \mathrm{m}$ LEKID array fabricated in Ref. 70 achieves a $400-\mu \mathrm{m}$ pitch with an area-efficient absorber/inductor design and small interdigitated capacitors. These small IDCs necessarily mean increasing the resonance frequency from $250 \mathrm{MHz}$ to $1.7 \mathrm{GHz}$. However, this is still less than the $\sim 5 \mathrm{GHz}$ frequency typical of CPW resonators and is consistent with the readout scheme of 2000 pixels per $4 \mathrm{GHz}$ band. While we do not believe they are required, parallel plate capacitors may be useful for these small pitch designs by adding further design flexibility.

\subsection{Cosmic Ray Mitigation}

Cosmic ray interactions with the detector substrate will produce high energy phonons, and the resulting glitches in the detector time streams can potentially impact a large fraction of the data. ${ }^{84}$ One way to mitigate this effect with KIDs is to deposit a low- $T_{c}$ film on the substrate, with the expectation that high energy phonons entering this film will quickly down-convert to phonons with energies near $2 \Delta_{0}$ (where $\Delta_{0}$ is the gap energy). At such low energies, these phonons will not be capable of breaking Cooper pairs in the KID and will not produce a detector response. , $^{2,55,85}$

Reference 55 demonstrated the effectiveness of this technique with laboratory measurements using the same type of array as in the SPACEKIDs program. They found that the dead time due to cosmic ray glitches could be reduced by a factor of 40 by depositing a layer of Ta on the backside (with $T_{c}=0.65 \mathrm{~K}$ compared with $T_{c}=1.25 \mathrm{~K}$ in the Al). Crucially, this work showed that the dead time per pixel was independent of array size, such that further increases in the array would not increase the data loss rate. When accounting for the high event rate in an L2 orbit, these authors predict a per-pixel dead time of $1.4 \%$. A comparable reduction in the dead time was found for devices in which the KID was placed on a thin membrane, isolating it from the larger substrate. In the SPACEKIDs demonstration array, a TiN mesh was employed to absorb stray light and act as a phonon trap, and the estimated dead time for an event rate appropriate for L2 was $4 \%{ }^{2}$

The OLIMPO balloon experiment conducted a stratospheric flight with arrays of lumped element KIDs operating at 150 to $460 \mathrm{GHz}$. As with the TIM prototypes, these KIDs use front-side illuminated, feedhorn-coupled absorbers with a metallized backshort. The backshort was made from $200 \mathrm{~nm}$ aluminum and doubles as a phonon trap (the $T_{c}$ is slightly less than that in the 30-nm aluminum film used to pattern the KIDs). An analysis of the data conducted in flight resulted in $<4 \%$ of the data contaminated by cosmic rays. ${ }^{44}$

\section{Summary}

The far-IR detectors for the FIP and OSS instruments on the Origins Space Telescope will need to achieve extremely low NEPs over the 25 to $588 \mu \mathrm{m}$ band and be deployed in kilopixel 
subarrays that may be suitably scaled up to arrays with $\sim 10^{4}$ detectors. We address the current status for KIDs to meet these requirements, focusing on the antenna-fed CPW devices developed in the SPACEKIDs program, as well as lumped element devices based on an extension of the detectors under development for the TIM balloon experiment. The SPACEKIDs detector array has achieved an NEP of $3 \times 10^{-19} \mathrm{~W} \mathrm{~Hz}^{-1 / 2}$, suitable for imaging with FIP but not yet reaching the NEP goal of $3 \times 10^{-20} \mathrm{~W} \mathrm{~Hz}^{-1 / 2}$ for spectroscopy with OSS. The lumped element TIM detectors have achieved an NEP of $4 \times 10^{-18} \mathrm{~W} \mathrm{~Hz}^{-1 / 2}$, and reducing this to $<1 \times 10^{-19} \mathrm{~W} \mathrm{~Hz}^{-1 / 2}$ should be possible with changes to the inductor/absorber designed to increase the responsivity. An array of lumped element KIDs designed to operate at $10 \mu \mathrm{m}$ has been fabricated and characterized, achieving a dark NEP of $\sim 4 \times 10^{-19} \mathrm{~W} \mathrm{~Hz}^{-1 / 2}$.

Kilopixel subarrays with sufficiently high multiplexing density have been demonstrated on ground-based telescopes, and this requirement should not pose a challenge for Origins. The SPACEKIDs and TIM detectors have both been demonstrated at $350 \mu \mathrm{m}$, but simulations suggest both architectures can be adapted to work down to $25 \mu \mathrm{m}$. The small $500 \mu \mathrm{m}$ pitch demanded by FIP and OSS represents a challenge for the SPACEKIDs CPW resonators, but a $400 \mu \mathrm{m}$ pitch has been demonstrated with the lumped element design. Cosmic ray contamination will be an issue for any detector architecture, but hardening KID arrays using low $T_{c}$ phonon traps promises to reduce the cosmic ray dead time to $<5 \%$.

\section{Acknowledgments}

Funding for this work was provided by the National Aeronautics and Space Administration (Grant No. 80NSSC19K0489). R. M. J. Janssen was supported by an appointment to the NASA Postdoctoral Program at the NASA Jet Propulsion Laboratory, administered by the Universities Space Research Association under contract with NASA. This research was carried out in part at the Jet Propulsion Laboratory, California Institute of Technology, under a contract with the National Aeronautics and Space Administration (80NM0018D0004). We thank the anonymous referees for many helpful comments on an earlier draft of this manuscript.

\section{References}

1. C. M. Bradford et al., "The origins survey spectrometer (OSS): a far-IR discovery machine for the Origins Space Telescope," Proc. SPIE 10698, 1069818 (2018).

2. J. Baselmans et al., "A kilo-pixel imaging system for future space based far-infrared observatories using microwave kinetic inductance detectors," Astron. Astrophys. 601, A89 (2017).

3. https://asd.gsfc.nasa.gov/firs/docs/OriginsVolume2TechDevelopmentPlanREDACTED.pdf.

4. P. Love et al., "1024 x 1024 Si: as IBC detector arrays for JWST MIRI," Proc. SPIE 5902, 590209 (2005).

5. G. Rieke et al., "The multiband imaging photometer for Spitzer (MIPS)," Astrophys. J. Suppl. Ser. 154(1), 25 (2004).

6. A. Poglitsch et al., "The photodetector array camera and spectrometer (PACS) on the Herschel space observatory," Astron. Astrophys. 518, L2 (2010).

7. J. Zmuidzinas and P. Richards, "Superconducting detectors and mixers for millimeter and submillimeter astrophysics," Proc. IEEE 92(10), 1597-1616 (2004).

8. P. Mauskopf, "Transition edge sensors and kinetic inductance detectors in astronomical instruments," Publ. Astron. Soc. Pac. 130(990), 082001 (2018).

9. D. Farrah et al., "Far-infrared instrumentation and technological development for the next decade," J. Astron. Telesc. Instrum. Syst. 5(2), 020901 (2019).

10. K. Irwin, "An application of electrothermal feedback for high resolution cryogenic particle detection," Appl. Phys. Lett. 66(15), 1998-2000 (1995).

11. A. Lee et al., "A superconducting bolometer with strong electrothermal feedback," Appl. Phys. Lett. 69(12), 1801-1803 (1996).

12. K. Irwin and G. Hilton, "Transition-edge sensors," in Cryogenic Particle Detection, Topics in Applied Physics, C. Enss, Ed., Vol. 99, pp. 63-150, Springer, Berlin, Heidelberg (2005). 
13. P. K. Day et al., "A broadband superconducting detector suitable for use in large arrays," Nature 425, 817-821 (2003).

14. J. Zmuidzinas, "Superconducting microresonators: physics and applications," Annu. Rev. Condens. Matter Phys. 3(1), 169-214 (2012).

15. D. Mattis and J. Bardeen, "Theory of the anomalous skin effect in normal and superconducting metals," Phys. Rev. 111(2), 412 (1958).

16. S. Doyle et al., "Lumped element kinetic inductance detectors," in Eighteenth Int. Symp. Space Terahertz Technol., A. Karpov, Ed., p. 170 (2007).

17. O. Noroozian, "Superconducting microwave resonator arrays for submillimeter/far-infrared imaging," PhD thesis, California Institute of Technology (2012).

18. L. J. Swenson et al., "MAKO: a pathfinder instrument for on-sky demonstration of low-cost 350 micron imaging arrays," Proc. SPIE 8452, 84520P (2012).

19. C. McKenney et al., "Design considerations for a background limited 350 micron pixel array using lumped element superconducting microresonators," Proc. SPIE 8452, 84520S (2012).

20. R. Duan et al., "An open-source readout for MKIDs," Proc. SPIE 7741, 77411V (2010).

21. J. Bueno et al., "Full characterisation of a background limited antenna coupled KID over an octave of bandwidth for THz radiation," Appl. Phys. Lett. 110, 233503 (2017).

22. J. Schlaerth et al., "A millimeter and submillimeter kinetic inductance detector camera," J. Low Temp. Phys. 151, 684-689 (2008).

23. J. Schlaerth et al., "MKID multicolor array status and results from DemoCam," Proc. SPIE 7741, 774109 (2010).

24. P. R. Maloney et al., "MUSIC for sub/millimeter astrophysics," Proc. SPIE 7741, 77410F (2010).

25. J. A. Schlaerth et al., "The status of music: a multicolor sub/millimeter MKID instrument," J. Low Temp. Phys. 167, 347-353 (2012).

26. J. Sayers et al., "The status of MUSIC: the multiwavelength sub-millimeter inductance camera," Proc. SPIE 9153, 915304 (2014).

27. A. Monfardini et al., "NIKA: a millimeter-wave kinetic inductance camera," Astron. Astrophys. 521, A29 (2010).

28. R. Adam et al., "The NIKA2 large-field-of-view millimetre continuum camera for the $30 \mathrm{~m}$ IRAM telescope," Astron. Astrophys. 609, A115 (2018).

29. A. Catalano et al., "The NIKA2 instrument at 30-m IRAM telescope: performance and results," J. Low Temp. Phys. 193, 916-922 (2018).

30. L. Perotto et al., "Calibration and performance of the NIKA2 camera at the IRAM 30-m telescope," Astron. Astrophys. 637, A71 (2020).

31. L. Otal, "The optical system and the astronomical potential of A-MKID, a new camera using microwave kinetic inductance detector technology," PhD Thesis, Rheinische FriedrichWilhelms-Universität Bonn (2015).

32. L. Ferrari et al., "MKID large format array testbed," IEEE Trans. Terahertz Sci. Technol. 8(6), 572-580 (2018).

33. G. Wilson et al., "The TolTEC project: a millimeter wavelength imaging polarimeter (Conference Presentation)," Proc. SPIE 10708, 107080I (2018).

34. J. E. Austermann et al., "Millimeter-wave polarimeters using kinetic inductance detectors for TolTEC and beyond," J. Low Temp. Phys. 193, 120-127 (2018).

35. "EAO submillimetre futures paper series," 2019, https://www.eaobservatory.org//jcmt/wpcontent/uploads/sites/2/2019/11/EAOSubmmFuturesWPs.pdf.

36. "A guide to the new JCMT $850 \mu \mathrm{m}$ MKID camera performance," 2019, https://www .eaobservatory.org//jcmt/wp-content/uploads/sites/2/2019/05/Guide-to-the-new-850umMKID-camera-performance.pdf.

37. W. Holland et al., "SCUBA-2: the 10,000 pixel bolometer camera on the James Clerk Maxwell Telescope," Mon. Not. R. Astron. Soc. 430(4), 2513-2533 (2013).

38. E. Kovetz et al., "Astrophysics and cosmology with line-intensity mapping," arXiv:1903.04496 (2019).

39. E. Shirokoff et al., "Design and performance of superspec: an on-chip, kid-based, mmwavelength spectrometer," J. Low Temp. Phys. 176(5-6), 657-662 (2014). 
40. T. Takekoshi et al., "DESHIMA on ASTE: on-sky responsivity calibration of the integrated superconducting spectrometer," J. Low Temp. Phys. 199, 231-239 (2020).

41. Concerto Collaborationet al., "A wide field-of-view low-resolution spectrometer at APEX: instrument design and scientific forecast," Astron. Astrophys. 642, A60 (2020).

42. J. Vieira et al., "The terahertz intensity mapper (TIM): an imaging spectrometer for galaxy evolution studies at high-redshift," in ISSTT2019, pp. 208-215, Gothenburg, Sweden (2019).

43. P. Ade et al., "The experiment for cryogenic large-aperture intensity mapping (EXCLAIM)," J. Low Temp. Phys. 199, 1027-1037 (2020).

44. S. Masi et al., "Kinetic inductance detectors for the OLIMPO experiment: in-flight operation and performance," J. Cosmol. Astropart. Phys. 2019, 003 (2019).

45. N. Lourie et al., "Preflight characterization of the BLAST-TNG receiver and detector arrays," Proc. SPIE 10708, 107080L (2018).

46. S. Rowe et al., "A passive terahertz video camera based on lumped element kinetic inductance detectors," Rev. Sci. Instrum. 87(3), 033105 (2016).

47. J. Sayers et al., "A millimeter-wave kinetic inductance detector camera for long-range imaging through optical obscurants," Proc. SPIE 11411, 114110H (2020).

48. S. Hailey-Dunsheath et al., "Development of aluminum LEKIDs for balloon-borne far-IR spectroscopy," J. Low Temp. Phys. 193, 968-975 (2018).

49. J. van Rantwijk et al., "Multiplexed readout for 1000-pixel arrays of microwave kinetic inductance detectors," IEEE Trans. Microwave Theory Tech. 64, 1876-1883 (2016).

50. P. J. de Visser et al., "Fluctuations in the electron system of a superconductor exposed to a photon flux," Nat. Commun. 5, 3130 (2014).

51. R. M. J. Janssen et al., "High optical efficiency and photon noise limited sensitivity of microwave kinetic inductance detectors using phase readout," Appl. Phys. Lett. 103, 203503 (2013).

52. R. Barends et al., "Noise in $\mathrm{NbTiN}, \mathrm{Al}$, and Ta superconducting resonators on silicon and sapphire substrates," IEEE Trans. Appl. Supercond. 19, 936-939 (2009).

53. J. Gao et al., "A semiempirical model for two-level system noise in superconducting microresonators," Appl. Phys. Lett. 92, 212504 (2008).

54. O. Noroozian et al., "Two-level system noise reduction for microwave kinetic inductance detectors," AIP Conf. Proc. 1185(1), 148-151 (2009).

55. K. Karatsu et al., "Mitigation of cosmic ray effect on microwave kinetic inductance detector arrays," Appl. Phys. Lett. 114, 032601 (2019).

56. R. Barends et al., "Reduced frequency noise in superconducting resonators," Appl. Phys. Lett. 97, 033507 (2010).

57. R. Nie et al., "Optimization of a quasi-mesh absorber for the terahertz intensity mapper," IEEE Trans. Terahertz Sci. Technol. 10, 704-712 (2020).

58. S. Kaplan et al., "Quasiparticle and phonon lifetimes in superconductors," Physical Review B 14(11), 4854 (1976).

59. R. Barends et al., "Quasiparticle relaxation in optically excited high-Q superconducting resonators," Phys. Rev. Lett. 100, 257002 (2008).

60. R. Barends et al., "Enhancement of quasiparticle recombination in Ta and Al superconductors by implantation of magnetic and nonmagnetic atoms," Phys. Rev. B 79, 020509 (2009).

61. P. J. de Visser et al., "Number fluctuations of sparse quasiparticles in a superconductor," Phys. Rev. Lett. 106, 167004 (2011).

62. H. McCarrick et al., "Horn-coupled, commercially-fabricated aluminum lumped-element kinetic inductance detectors for millimeter wavelengths," Rev. Sci. Instrum. 85(12), 123117 (2014).

63. A. Fyhrie et al., "Decay times of optical pulses for aluminum cpw kids," J. Low Temp. Phys. 199, 688-695 (2020).

64. J. Hubmayr et al., "Photon-noise limited sensitivity in titanium nitride kinetic inductance detectors," Appl. Phys. Lett. 106, 073505 (2015).

65. S. Kumar et al., "Temperature dependence of the frequency and noise of superconducting coplanar waveguide resonators," Appl. Phys. Lett. 92, 123503 (2008).

66. L. J. Swenson et al., "Operation of a titanium nitride superconducting microresonator detector in the nonlinear regime," J. Appl. Phys. 113, 104501 (2013). 
67. http://thz.asu.edu/products.html.

68. P. J. de Visser et al., "Evidence of a nonequilibrium distribution of quasiparticles in the microwave response of a superconducting aluminum resonator," Phys. Rev. Lett. 112, 047004 (2014).

69. D. J. Goldie and S. Withington, "Non-equilibrium superconductivity in quantum-sensing superconducting resonators," Supercond. Sci. Technol. 26, 015004 (2013).

70. J. Perido et al., "Extending kids to the mid-IR for future space and suborbital observatories," J. Low Temp. Phys. 199, 696-703 (2020).

71. J. Glenn et al., "Galaxy Evolution Probe concept study report," 2019, https://smd-prod.s3 .amazonaws.com/science-red/s3fs-public/atoms/files/GEP_Study_Rpt.pdf.

72. creativecommons.org/licenses/by/4.0/legalcode.

73. F. Defrance et al., "A 1.6:1 bandwidth two-layer antireflection structure for silicon matched to the 190-310 GHz atmospheric window," Appl. Opt. 57(18), 5196-5209 (2018).

74. P. Savander, "Microlens arrays etched in glass and silicon," Opt. Lasers Eng. 20, 97-107 (1994).

75. K. P. Larsen, J. T. Ravnkilde, and O. Hansen, "Investigations of the isotropic etch of an ICP source for silicon microlens mold fabrication," J. Micromech. Microeng. 15, 873-882 (2005).

76. C.-F. Chen et al., "Silicon microlens structures fabricated by scanning-probe gray-scale oxidation," Opt. Lett. 30, 652-654 (2005).

77. P. N. A. Belmonte et al., "Microfabrication and characterization of single-mask silicon microlens arrays for the IR spectra," Proc. SPIE 9130, 91300D (2014).

78. Z. Deng et al., "Fabrication of large-area concave microlens array on silicon by femtosecond laser micromachining," Opt. Lett. 40, 1928-1931 (2015).

79. X. Meng et al., "Simple fabrication of closed-packed IR microlens arrays on silicon by femtosecond laser wet etching," Appl. Phys. A 121, 157-162 (2015).

80. H. Zuo et al., "CMOS compatible fabrication of micro, nano convex silicon lens arrays by conformal chemical vapor deposition," Opt. Express 25, 3069-3076 (2017).

81. J. F. Gonzàlez et al., "Infrared antennas coupled to lithographic Fresnel zone plate lenses," Appl. Opt. 43, 6067-6073 (2004).

82. X. Liu et al., "Superconducting micro-resonator arrays with ideal frequency spacing," Appl. Phys. Lett. 111(25), 252601 (2017).

83. S. Shu et al., "Increased multiplexing of superconducting microresonator arrays by postcharacterization adaptation of the on-chip capacitors," Appl. Phys. Lett. 113, 082603 (2018).

84. Planck Collaboration et al., "Planck 2013 results. X. HFI energetic particle effects: characterization, removal, and simulation," Astron. Astrophys. 571, A10 (2014).

85. A. Monfardini et al., "Lumped element kinetic inductance detectors for space applications," Proc. SPIE 9914, 99140N (2016).

Biographies of the authors are not available. 